\title{
Low-intensity pulsed ultrasound affects human articular chondrocytes in vitro
}

\author{
C. M. Korstjens $\cdot$ R. H. H. van der Rijt • \\ G. H. R. Albers $\cdot$ C. M. Semeins $\cdot$ J. Klein-Nulend
}

Received: 14 January 2008 / Accepted: 8 July 2008/Published online: 14 October 2008

(c) The Author(s) 2008. This article is published with open access at Springerlink.com

\begin{abstract}
We investigated whether low-intensity pulsed ultrasound (LIPUS) stimulates chondrocyte proliferation and matrix production in explants of human articular cartilage obtained from donors suffering from unicompartimental osteoarthritis of the knee, as well as in isolated human chondrocytes in vitro. Chondrocytes and explants were exposed to LIPUS $\left(30 \mathrm{~mW} / \mathrm{cm}^{2} ; 20 \mathrm{~min} /\right.$ day, 6 days). Stimulation of $\left[{ }^{35} \mathrm{~S}\right]$-sulphate incorporation into proteoglycans by LIPUS was 1.3 -fold higher in degenerative than in collateral monolayers as assessed biochemically and 1.9-fold higher in explants as assessed by autoradiography. LIPUS decreased the number of cell nests containing 1-3 chondrocytes by 1.5 fold in collateral and by 1.6 fold in degenerative explants. LIPUS increased the number of nests containing $4-6$ chondrocytes by 4.8 fold in collateral and by 3.9 fold in degenerative explants. This suggests that LIPUS stimulates chondrocyte proliferation and matrix production in chondrocytes of human articular cartilage in vitro. LIPUS might provide a feasible tool for cartilage tissue repair in osteoarthritic patients, since it stimulates chondrocyte proliferation and matrix production.
\end{abstract}

C. M. Korstjens and R. H. H. van der Rijt are first authors.

C. M. Korstjens $(\square) \cdot$ C. M. Semeins · J. Klein-Nulend Department of Oral Cell Biology, ACTA-University of Amsterdam and VU University Amsterdam, Van der Boechorststraat 7, 1081 BT Amsterdam, The Netherlands

e-mail: c.korstjens@vumc.nl

R. H. H. van der Rijt - G. H. R. Albers Department of Orthopedics, Tergooiziekenhuizen Hilversum, PO Box 10016, 1201 DA Hilversum, The Netherlands
Keywords Low intensity pulsed ultrasound (LIPUS) . Osteoarthritis · Articular cartilage .

Chondrocyte proliferation · Proteoglycans

\section{Introduction}

Articular cartilage has a very limited capacity for selfrepair [6]. Traumatic chondral lesions often result in chronic degeneration in the long run, and eventually osteoarthritis (OA) can develop [6]. The long-term prognosis of these chondral lesions is of eminent importance, especially in adults aged less than 50 years, since arthroplasty is not an option to consider at younger age. These patients will likely benefit from a treatment that improves the repair of chondral tissue. Such a treatment could postpone the need for surgical treatment or even make surgical treatment in a later stage unnecessary.

Low-intensity pulsed ultrasound (LIPUS) has been shown to stimulate osteogenesis and fracture healing $[5,6$, $11,14,15,18,19]$. LIPUS not only stimulates bone formation in vitro as well as in vivo [5, 10, 11, 14, 15, 18], but it also exerts anabolic effects on cartilage formation $[2,13$, 16, 17, 20-22]. The stimulatory effect of LIPUS on chondrocytes and cartilage matrix production in fractured bone results in stimulation of the healing process and improves the repair of chondral tissue [2, 12, 16, 17, 20]. LIPUS has been reported to stimulate rat chondrocyte proliferation and matrix production in vitro, suggesting an overall anabolic effect on cartilage formation [16, 17, 20]. LIPUS also stimulated the healing of osteochondral defects in rabbit knees in vivo [2]. Whether LIPUS also exerts an anabolic effect on human osteoarthritic or non-osteoarthritc chondrocyte proliferation and cartilage matrix production is as yet unknown. Therefore, we investigated whether 
LIPUS stimulates proliferation and matrix production of chondrocytes in explants from human articular cartilage, obtained from the degenerative compartment and the collateral compartment from donors suffering from unicompartimental $\mathrm{OA}$ of the knee, as well as in isolated human chondrocytes in vitro.

\section{Materials and methods}

\subsection{Culture of chondrocytes}

Human articular cartilage samples (surgical waste) were harvested during total knee arthroplasty from two male donors, aged 69 and 71 years, suffering from unicompartimental OA of the knee. The protocol was approved by the Ethical Review Board of the Vrije Universiteit Medical Center. The cells of the collateral compartment of one donor are the controls for the cells of the degenerative compartment of the same donor. Therefore, this is a within donor-controlled study. The cartilage from the degenerative compartment and the collateral compartment were kept separately. The articular cartilage was kept under sterile conditions in phosphate buffered saline (PBS) for maximally $3 \mathrm{~h}$, until it was separated from the underlying bone. Successively the cartilage was morsalized to roughly $1 \mathrm{~mm}$ pieces and incubated with $2 \mathrm{mg} / \mathrm{ml}$ collagenase II (Worthington, Freehold, NJ, USA) in Dulbecco's Modified Eagle Medium (DMEM, Gibco, Paisley, UK) for $2 \mathrm{~h}$ at $37^{\circ} \mathrm{C}$. The released chondrocytes were cultured in six-well culture plates (Costar, Cambridge, MA, USA) at $25 \times 10^{3}$ cells/well with $2.54 \mathrm{ml}$ of fluid medium per well. One culture plate per donor was used for chondrocytes obtained from the degenerative knee compartment, and one plate per donor for chondrocytes obtained from the collateral compartment. In total, four culture plates were collected for this study. The culture medium consisted of DMEM (Gibco) without nucleosides, supplemented with $10 \%$ fetal bovine serum (FBS, Hyclone, Logan, UT, USA), $0.6 \mathrm{mM} \mathrm{L}(+)$ ascorbic acid (Merck, Darmstadt, Germany), $1.25 \mu \mathrm{g} / \mathrm{ml}$ fungizone (Gibco), $50 \mu \mathrm{g} / \mathrm{ml}$ gentamycin (Gibco), $100 \mathrm{U} /$ $\mathrm{ml}$ penicillin (Sigma, St Louis, MO, USA) and $50 \mu \mathrm{g} / \mathrm{ml}$ streptomycin sulphate (Sigma). Culture medium was changed twice per week and before the start of LIPUS treatment. Before $\left[{ }^{35} \mathrm{~S}\right]$-sulphate labeling as described below, the medium was replaced as well. The culture plates were placed in a $37^{\circ} \mathrm{C}$ incubator containing a humidified atmosphere with $5 \% \mathrm{CO}_{2}$ in air and cultured for 7 days to allow formation of chondrocyte monolayer cultures. LIPUS treatment was started after 7 days of pre-culture, as described below.

\subsection{Culture of cartilage explants}

An explant model was used, in which the chondrocytes reside within their natural environment, surrounded by their own matrix. By using this in vitro explant model, we aimed to mimic the in vivo situation as closely as possible. Full thickness cartilage samples (surgical waste) were taken from 4 donors, 1 male and 3 females, age 6172 years, with unicompartimental OA of the knee. The protocol was approved by the Ethical Review Board of the Vrije Universiteit Medical Center. The explants of the collateral compartment of one donor are the controls for the explants of the degenerative compartment of the same donor. Therefore, this is a within donor-controlled study. Articular cartilage was collected from harvested waste material during total knee arthroplasty from the degenerative as well as from the collateral compartment. At the day of surgery, cylindrical full thickness cartilage explants were taken from the weight-bearing surface, by using an $10 \mathrm{~cm} 11$ gauge diameter biopsy needle (Osteo-Site ${ }^{\circledR}$ Bone Biopsy Needle, Cook Medical Inc., Bloomington, IL, USA). The cartilage explants were kept under sterile conditions in PBS, which was replaced by culture medium within $3 \mathrm{~h}$.

The explants were separated from the underlying bone and cultured in six-well culture plates (Costar) in $2.54 \mathrm{ml}$ of fluid medium per well, with one explant per well. The medium consisted of DMEM (Gibco) without nucleosides, supplemented with 10\% FBS (Hyclone), $0.6 \mathrm{mM} \mathrm{L}(+)$ ascorbic acid (Merck), $1.25 \mu \mathrm{g} / \mathrm{ml}$ fungizone (Gibco), $50 \mu \mathrm{g} / \mathrm{ml}$ gentamycin (Gibco), $100 \mathrm{U} / \mathrm{ml}$ penicillin (Sigma) and $50 \mu \mathrm{g} / \mathrm{ml}$ streptomycin sulphate (Sigma). Culture medium was changed twice per week and before the start of LIPUS treatment. Before $\left[{ }^{35} \mathrm{~S}\right]$ sulphate labeling as described below, the medium was replaced as well. The explant cultures were placed in a $37^{\circ} \mathrm{C}$ incubator containing a humidified atmosphere with $5 \% \mathrm{CO}_{2}$ in air and cultured. LIPUS treatment was started after $24 \mathrm{~h}$ of preculture, as described below.

\subsection{Low intensity pulsed ultrasound (LIPUS) treatment}

LIPUS treatment was started after 7 days of pre-culture for the monolayer cultures, and after $24 \mathrm{~h}$ of pre-culture for the cartilage explants. Half of the degenerative chondrocyte monolayers and cartilage explant cultures and half of the collateral chondrocyte monolayers and cartilage explant cultures in the six-well culture plates were exposed to LIPUS $\left(30 \mathrm{~mW} / \mathrm{cm}^{2}\right)$ during $20 \mathrm{~min}$ per day for a period of 6 days. The other half of the cultures served as non-treated controls. The therapy unit consisted of two sonic accelerated fracture healing system devices (SAFHS ${ }^{\circledR}$, model 2A; 
Exogen, Smith \& Nephew Inc., Memphis, TN, USA) and three transducers per device, which were connected by coupling gel to the six-well culture plate. The wells were filled with $2.54 \mathrm{ml}$ standardized fluid culture medium to achieve a liquid-height equivalent to one quarter of the carrier frequency wavelength [15]. The SAFHS ${ }^{\circledR}$ device provides low-intensity ultrasound at $1.5 \mathrm{MHz}$ frequency. This setting was used in earlier experiments by Smith and Nephew Inc. and has proven to provide ultrasonic waves in the medium [15]. The distance between the transducer and the cultures was less than $2 \mathrm{~mm}$. Control culture plates were kept under identical conditions in six well plates, but were not exposed to LIPUS treatment.

\section{$2.4\left[{ }^{35} \mathrm{~S}\right]$-Labeling and biochemical analysis}

To assess cartilage formation biochemically, $\left[{ }^{35} \mathrm{~S}\right]$-sulphate incorporation into proteoglycans was measured. The monolayers and the explant cultures were labeled with $\left[{ }^{35} \mathrm{~S}\right]$-sulphate by pulsing with $10 \mu \mathrm{l}$ and $20 \mu \mathrm{l}$ $\mathrm{Na}_{2}{ }^{35} \mathrm{SO}_{4}$ (specific activity $1000 \mathrm{Ci} / \mathrm{mMole}$; Radiochemical Centre, Amersham, UK) for the last $24 \mathrm{~h}$ of culture. After removal of the medium, the monolayer cultures were rinsed extensively with PBS to remove exchangeable radioactivity [8], and incubated for $1 \mathrm{~h}$ with $0.5 \mathrm{M}$ $\mathrm{NaOH}$. The explants were rinsed three times extensively with PBS to remove exchangeable radioactivity [8], and $0.5 \mathrm{ml} 6 \mathrm{M} \mathrm{HCl}$ was added. The $\left[{ }^{35} \mathrm{~S}\right]$-sulphate incorporation into proteoglycans was quantified by liquid scintillation counting (LKB-Wallac, Rockville, MD, USA).

To normalize $\left[{ }^{35} \mathrm{~S}\right]$-sulphate incorporation for the monolayer cultures, the DNA content was determined by using CyQuant cell proliferation assay kit (C-7026) (Molecular Probes, Invitrogen, Carlsbad, CA, USA). DNA content was quantified by measuring fluorescence at $520 \mathrm{~nm}$ using a Fluostar Galaxy spectrophotometer (BMG labtechnologies, Offenburg, Germany). Since the matrix components of the explants interfere with the DNA measurements, the $\left[{ }^{35} \mathrm{~S}\right]$-sulphate incorporation was expressed per milligram dry weight.

\subsection{Autoradiography}

Autoradiography was performed on 16 explants. After $\left[{ }^{35} \mathrm{~S}\right]$-sulphate labeling of proteoglycans, the cultured explants were fixed in $4 \%$ phosphate-buffered formaldehyde (Merck) overnight at $4^{\circ} \mathrm{C}$, and subsequently embedded in glycol methacrylate (GMA). For every explant, three undecalcified sections of $5 \mu \mathrm{m}$ thickness in the center of the explant were cut by a heavy duty microtome (Jung-K, R. Jung, Heidelberg, Germany). The distance between the three sections was $10 \mu \mathrm{m}$. $\left[{ }^{35} \mathrm{~S}\right]$-Sulphate incorporation in the matrix by chondrocytes was analysed by autoradiography performed with Ilford Nuclear Emulsion (Ilford, Essex, UK). The exposure time was 3.5 weeks, as determined by the amount of $\left[{ }^{35} \mathrm{~S}\right]$-sulphate incorporated. The sections were counterstained with toluidine blue. $\left[{ }^{35} \mathrm{~S}\right]$-Sulphate incorporation was assessed by counting the number of dots per cell in the complete microscopic field. This is indicative for the amount of $\left[{ }^{35} \mathrm{~S}\right]$-sulphate incorporated into proteoglycans during cartilage matrix production.

\subsection{Histology}

The cartilage explants that were not labeled with $\left[{ }^{35} \mathrm{~S}\right]-$ sulphate were used for histology. After fixation in $10 \%$ phosphate-buffered formalin overnight at $4^{\circ} \mathrm{C}$, the samples were embedded in methylmetacrylate (MMA). Undecalcified sections of $5 \mu \mathrm{m}$ thickness were cut by a heavy duty microtome (Jung-K). Sections were stained with $0.1 \%$ toluidin blue for light microscopy and histomorphometry. To monitor proliferation in the center of the explants, the number of cell nests and the size of the cell nests were assessed at two locations: (1) in the superficial layer, which is the layer closest to the joint, and (2) in the deep layer, which is the layer closest to the bone.

\subsection{Statistical methods}

A power analysis for the number of cultures and for the number of explants was conducted to calculate the sample size needed. Using a two-sided significance level of $5 \%$ and a power of $60 \%$ a sample size of $n=6$ per group is sufficient to be able to detect an effect size of $d=1.40$ $[d=($ mean $1-$ mean2/SD $)][1]$.

Data are expressed as mean \pm SEM. Twenty-four cultures were obtained for the monolayer cultures, 64 explants were obtained for the explant cultures, 16 explants were obtained for autoradiography, and 64 explants were obtained for histology. Statistical analysis was performed using Student's $t$ test. A $P$ value less than 0.05 was considered statistically significant.

\section{Results}

\section{1 $\left[{ }^{35} \mathrm{~S}\right]$-Sulphate incorporation in monolayer cultures}

LIPUS treatment stimulated $\left[{ }^{35} \mathrm{~S}\right]$-sulphate incorporation in both the degenerative and collateral monolayer cultures by respectively 1.5 -fold $(P<0.05)$ and 1.2 -fold $(P<0.05)$ (Fig. 1). Stimulation of $\left[{ }^{35} \mathrm{~S}\right]$-sulphate incorporation by 


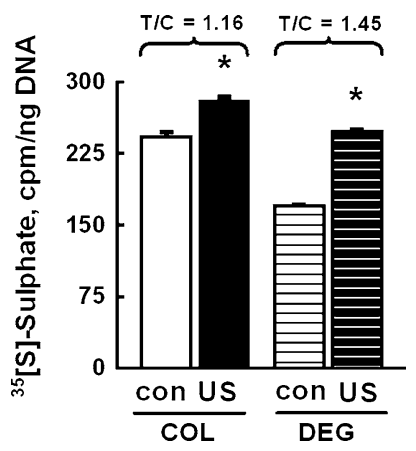

Fig. 1 Effect of LIPUS on ${ }^{35}[\mathrm{~S}]$-sulphate incorporation into glycosaminoglycans of degenerative and collateral human chondrocyte monolayer cultures. Results are mean \pm SEM of 24 chondrocyte cultures obtained from 2 donors. T/C LIPUS-treated-over-control ratio, $C O L$ chondrocytes obtained from the collateral compartment, $D E G$ chondrocytes obtained from the degenerative compartment, US LIPUS treated, con untreated control. * Significant difference in ${ }^{35}[\mathrm{~S}]$-sulphate incorporation $(P<0.05)$

LIPUS was 1.3-fold higher in the degenerative than in the collateral monolayers (Fig. 1).

\section{$3.2\left[{ }^{35} \mathrm{~S}\right]$-Sulphate incorporation in explants}

$\left[{ }^{35} \mathrm{~S}\right]$-Sulphate incorporation was 1.2-fold higher $(P=0.043)$ in untreated degenerative explants in comparison with untreated collateral explants (Fig. 2). $\left[{ }^{35} \mathrm{~S}\right]-$ Sulphate incorporation was also increased in the LIPUStreated degenerative explants compared to the LIPUS-treated collateral explants, but no statistical significance $(P=0.073)$ was reached (Fig. 2). LIPUS treatment did not result in changes in $\left[{ }^{35} \mathrm{~S}\right]$-sulphate incorporation in the degenerative and collateral explants in comparison with the untreated control cartilage explants (control collateral

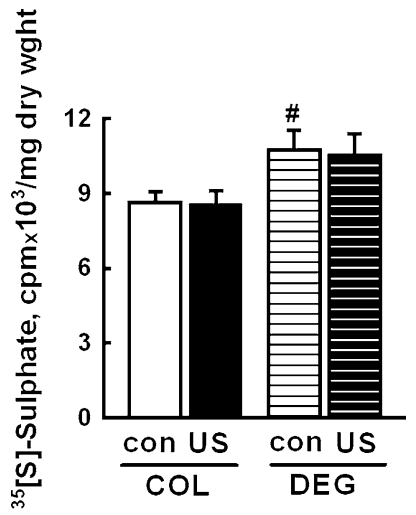

Fig. $2{ }^{35}[\mathrm{~S}]$-Sulphate incorporation into glycosaminoglycans in human cartilage explants of degenerative and collateral explants. Results are mean \pm SEM of 64 explants obtained from four donors. $C O L$ explants obtained from the collateral compartment, $D E G$ explants obtained from the degenerative compartment, US LIPUS treated, con untreated control. \# Significant difference in ${ }^{35}[\mathrm{~S}]-$ sulphate incorporation between untreated degenerative explants and untreated collateral explants $(P=0.043)$

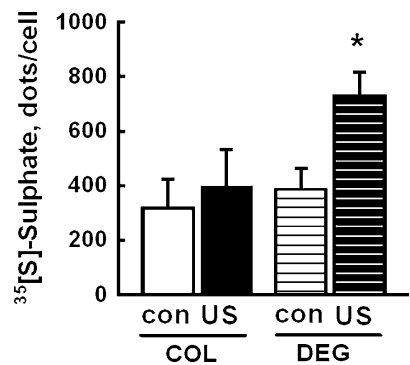

Fig. 3 Effect of LIPUS on ${ }^{35}[\mathrm{~S}]$-sulphate into glycosaminoglycans in human cartilage explants of degenerative and collateral explants analysed by autoradiography. Results are mean \pm SEM dots per cell of 16 explants obtained from one donor. $C O L$ explants obtained from the collateral compartment, $D E G$ explants obtained from the degenerative compartment, US LIPUS treated, con untreated control. * Significant effect of LIPUS in degenerative explants $(P=0.03)$

$8.6 \pm 0.4 \mathrm{cpm} \times 10^{3} / \mathrm{mg}$ dry weight; LIPUS-treated collateral $8.6 \pm 0.5 \mathrm{cpm} \times 10^{3} / \mathrm{mg}$ dry weight; control degenerative $10.7 \pm 0.8 \mathrm{cpm} \times 10^{3} / \mathrm{mg}$ dry weight; LIPUS-treated degenerative $10.5 \pm 0.9 \mathrm{cpm} \times 10^{3} / \mathrm{mg}$ dry weight; values are mean \pm SEM).

\subsection{Autoradiography of explants}

LIPUS resulted in a significant increase in the number of dots per cell in its surrounding matrix by 1.9 -fold in degenerative cartilage explants compared to control cartilage explants $(P=0.03)$ (Fig. 3). In the collateral explants a similar trend of a higher increase (1.3-fold) in the number of dots per cell in its surrounding matrix in the LIPUStreated cartilage explants was found in comparison with the untreated control cartilage explants (Fig. 3).

\subsection{Histomorphometry of explants}

Detailed photographs of the superficial and deep layer of an explant treated with LIPUS and an untreated control explant are shown in Fig. 4.

In the deep layer of the cartilage explants, LIPUS treatment decreased the number of cell nests containing 13 cells significantly in both the collateral explants by 1.5 fold $(P=0.009)$ and in the degenerative explants by 1.6fold $(P=0.05)$ (Fig. 5). However, LIPUS treatment did not decrease the number of cell nests containing 1-3 cells in the superficial layer of the collateral explants nor of the degenerative explants. In the superficial layer of the collateral explants, LIPUS treatment significantly stimulated the number of nests containing $4-6$ cells by 6.5 -fold $(P=0.006)$ (Fig. 6). LIPUS treatment did not change the number of nests containing 4-6 cells in the degenerative explants in the superficial layer. In the deep layer, LIPUS treatment stimulated the number of nests containing 4-6 cells significantly by 4.8 -fold $(P<0.001)$ in the collateral 
Fig. 4 Histology of human cartilage explants obtained from the collateral compartment. Details of the superficial layer (a) and deep layer (b) of untreated control cartilage explants, and of the superficial layer (c) and deep layer (d) of LIPUS treated cartilage explants. In the deep layer the cell nests (arrows) containing more cells. Original magnification $200 \times$, toluidin blue stained
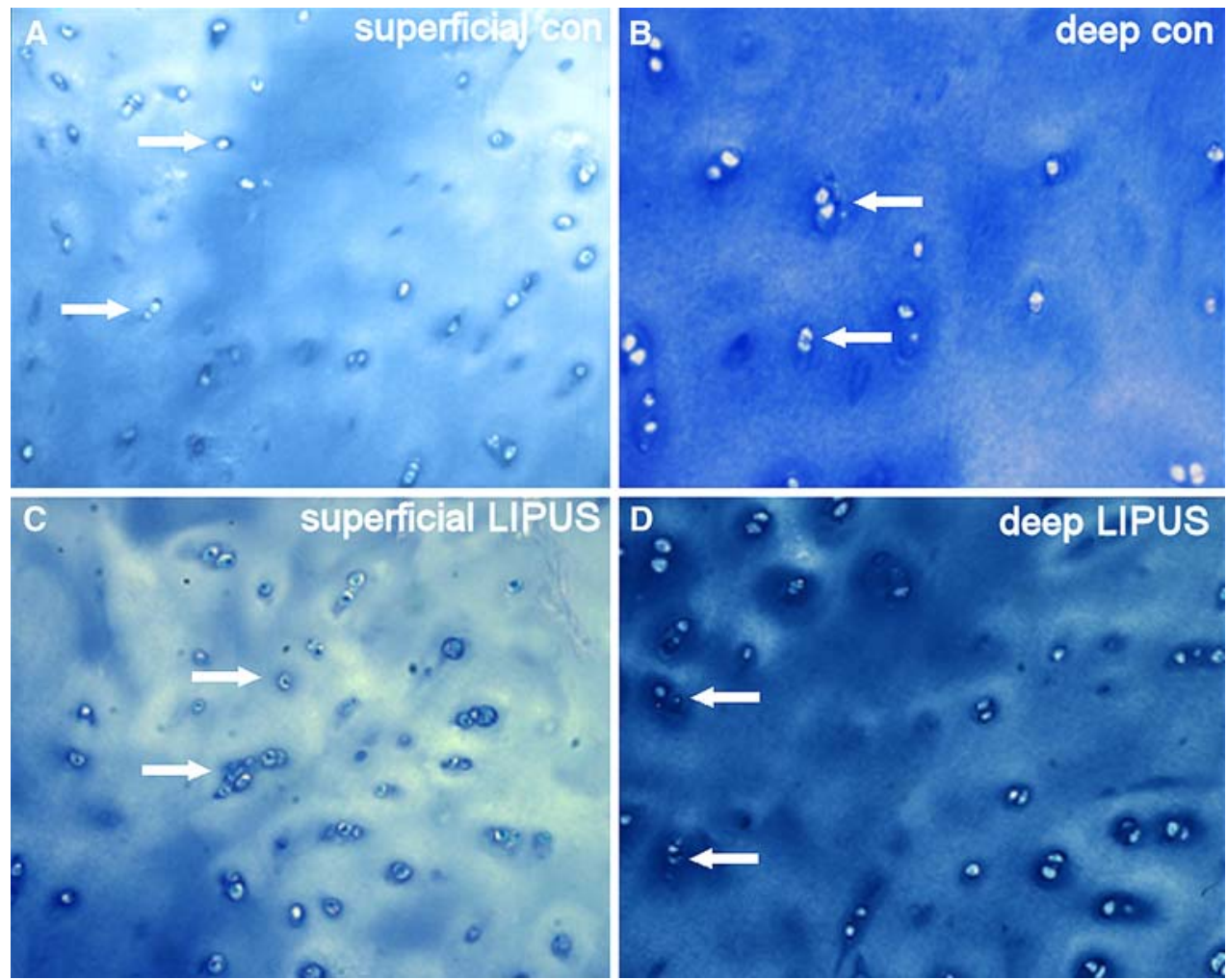

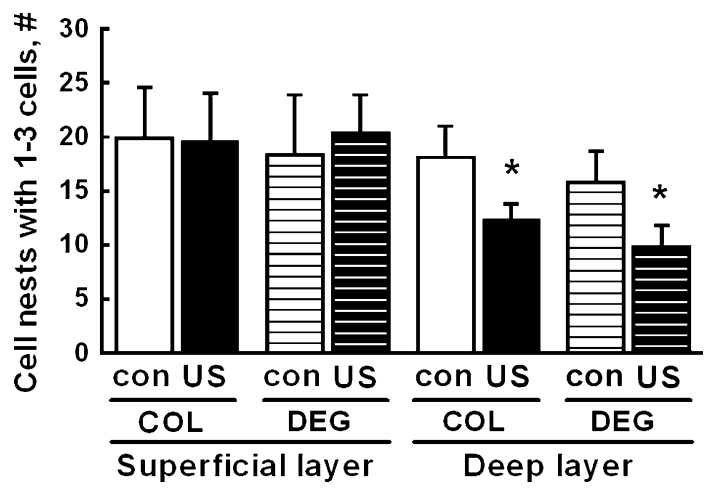

Fig. 5 Effect of LIPUS on number of cell-nests in the superficial layer (closest to the joint) and the deep layer (closest to the bone) in human degenerative and collateral cartilage explants. Results are mean \pm SEM of the number of cell nests with 1-3 cells-per-nest of 64 explants obtained from four donors. $C O L$ explants obtained from the collateral compartment, $D E G$ explants obtained from the degenerative compartment; con untreated control, US LIPUS treated. * Significant effect of LIPUS in the deep layer of degenerative $(P=0.05)$ and collateral $(P=0.009)$ explants

explants and by 3.9-fold in the degenerative explants $(P<0.001)$ (Fig. 6).

\section{Discussion}

In this study, we have investigated the effect of lowintensity pulsed ultrasound (LIPUS) on human chondrocyte

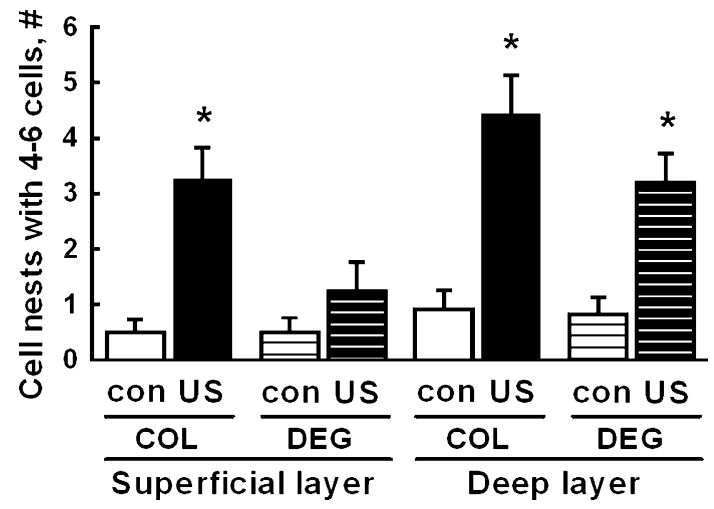

Fig. 6 Effect of LIPUS on number of cell-nests in the superficial layer (closest to the joint) and the deep layer (closest to the bone) in human degenerative and collateral cartilage explants. Results are mean \pm SEM of the number of cell nests with 4-6 cells-per-nest of 64 explants obtained from four donors. $C O L$ explants obtained from the collateral compartment, $D E G$ explants obtained from the degenerative compartment, con untreated control, US LIPUS treated. * Significant effect of LIPUS in the superficial layer collateral explants $(P=0.006) .{ }^{*}$ Significant effect of LIPUS in the deep layer of degenerative and collateral explants $(P<0.001)$

proliferation and cartilage matrix production in vitro. We hypothesized that LIPUS stimulates both chondrocyte proliferation and matrix production in human degenerative and collateral cartilage explants as well as in cultures of isolated human chondrocytes. We are aware that that not only the degenerative compartment but also the collateral 
compartment of the degenerative joint might be involved in the degenerative process. Surgeons have diagnosed unicompartimental OA of the knee in the donors, according to general accepted criteria [7]. The severity of the symptoms determines the need for treatment at the degenerative site [7]. The biopsy in the collateral compartment was taken where the cartilage appeared intact. We found differences in chondrocyte proliferation and matrix production after LIPUS stimulation in the degenerative and collateral compartments. This gives us reliance that our study design is appropriate to investigate whether LIPUS exerts an anabolic effect on human osteoarthritic or non-osteoarthritc (or less osteoarthritic) chondrocyte proliferation and cartilage matrix production.

We were able to harvest sufficient cartilage from two donors in order to establish monolayer cultures. The amount of cartilage that could be harvested from the other donors was too limited to establish monolayer cultures. A sufficient amount of cartilage explants could be obtained from four donors. The data suggest that LIPUS stimulates cartilage matrix production in both degenerative and collateral monolayer cultures and human explants and stimulates chondrocyte proliferation in both degenerative and collateral human explants. This is in agreement with chondrogenic stimulatory effects found by LIPUS treatment in chick embryos, rats and rabbits $[2,16,17,20,21]$.

In chondrocyte monolayer cultures a significant stimulatory effect of LIPUS treatment on $\left[{ }^{35} \mathrm{~S}\right]$-sulphate incorporation was observed, especially in degenerative chondrocytes. $\left[{ }^{35} \mathrm{~S}\right]$-Sulphate incorporation was significantly increased in degenerative explants compared to collateral explants for the untreated control explants. LIPUS-treated explants showed comparable increase in sulphate incorporation in the degenerative explants compared to the collateral explants, but reached no statistical significance. LIPUS did not result in overall significant changes in $\left[{ }^{35} \mathrm{~S}\right]$-sulphate incorporation compared to the untreated control explants in neither degenerative explants nor collateral explants using biochemical analysis suggesting that LIPUS did not affect matrix turnover. However, increased $\left[{ }^{35} \mathrm{~S}\right]$-sulphate incorporation in LIPUStreated degenerative explants of one donor was seen, indicating a stimulation of matrix production resulting from LIPUS treatment in the explants of this single donor. This suggests differences between cartilage obtained from one donor and cartilage obtained from another donor. This finding is comparable to the results obtained by KopakkalaTani et al. [9] who found that chondrocytes obtained from one donor out of five donors remained non-responsive to ultrasound treatment. Several studies have addressed the role of LIPUS as a stimulus for aggrecan synthesis in relation to chondrogenesis $[4,16,20,21]$. LIPUS stimulated aggrecan mRNA expression and proteoglycan synthesis in rat chondrocytes, and in a rat femur fracture model LIPUS increased mechanical properties of the healing fracture by accelerating synthesis of extra cellular matrix proteins $[16,20]$. Our finding that LIPUS stimulates matrix production in articular cartilage monolayer cultures is in agreement with these studies. In addition, TGF- $\beta$ treatment of mesenchymal stem cells has been shown to enhance chondrogenic differentiation, which is related to an increase in aggrecan deposition [4]. Application of LIPUS for 20 min per day to the TGF- $\beta$ treated mesenchymal stem cells even further increased aggregan deposition [4]. LIPUS enhanced chondrogenic differentiation, but not cell proliferation [4]. In an aggregate chondrocyte culture system LIPUS promotes proliferation, and retains the differentiation state of chondrocytes [12]. The effect of LIPUS on proliferation and differentiation was found to be mediated by TGF- $\beta$ [12].

Different types of cartilage, i.e. hyaline cartilage and endochondral cartilage, which transforms into bone, show different reactions to LIPUS stimulation [21]. LIPUS enhances bone formation by stimulating hypertrophy of chondrocytes directed to terminal differentiation, but it does not induce hypertrophy of chondrocytes in hyaline cartilage [21]. LIPUS stimulation does not affect tissue maturation in mice with hyaline-like cartilage implants (generated in vitro) implanted subcutaneously in the back, suggesting that LIPUS treatment does not accelerate maturation of tissue-engineered neo-cartilage [3].

Although the number per group for the autoradiography experiment was low ( $n=4$ per group), we found an effect size of $d>2$ [1]. This means that the power is much higher than $60 \%$ and that the findings are of importance. Our observation of increased $\left[{ }^{35} \mathrm{~S}\right]$-sulphate incorporation in proteoglycans by autoradiography suggests a stimulatory effect of LIPUS on chondrogenesis. In degenerative explants increased proteoglycan synthesis was found compared to collateral explants, suggesting a stimulation of cartilage matrix production by individual cells. These results are in agreement with reported effects of LIPUS on hyaline cartilage in the distal part of the sternum of chick embryos [21]. The sternum of chick embryos is characterized by a proximal part that shows endochondral ossification and a hyaline distal part that does not calcify. In the proximal part LIPUS may augment bone formation by stimulating hypertrophy of chondrocytes that are developing toward terminal differentiation; in the distal part LIPUS induces increased type II collagen and aggrecan production, indicating a potential role in cartilage repair [21].

Histomorphometric analysis showed that LIPUS stimulation significantly decreased the number of nests with a low number of chondrocytes (1-3 cells per nest) for the degenerative and for the collateral explants in the deep cartilage layer. The number of cell nests with high 
chondrocyte numbers (4-6 cells per nest) was strongly increased in the degenerative and in the collateral explants in the deep cartilage layer, suggesting that LIPUS stimulates chondrocyte proliferation in the deep layer of cartilage. Since the stimulation of cell nests with high chondrocyte numbers (4-6), the number of nests with low numbers of chondrocytes (1-3) was significantly reduced. These findings are interesting since cellular differences might underlie differences in cartilage quality. LIPUS evidently stimulates chondrocyte proliferation in the deep cartilage layer of human explants, layer closest to the bone, which normally contains less cells than the superficial layer, layer closest to the joint. The effects of LIPUS treatment on the number of cells in a cell nest are seen in degenerative cartilage as well as in collateral cartilage. LIPUS-treated chondrocytes in hyaline cartilage of the distal part of the sternum of chick embryos has been shown an intensity dependent proliferation and a decreased hypertrophic differentiation, which may influence the restoration and repair of articular cartilage [22]. However, Nishikori et al. [13] found that LIPUS treated in gel embedded chondrocytes of articular cartilage of 10 week old rabbits did not show stimulated cell proliferation, but an increase in chondroitin sulphate synthesis. Since we studied cartilage explants instead of gel embedded chondrocytes, our results, indicating an increase in number of cell nests and cell-nest-size in human chondrocytes, are not in agreement with the observations Nishikori et al. [13].

In conclusion, the present study suggests that LIPUS stimulates chondrocyte proliferation and matrix production in cartilage obtained from the degenerative compartment and to a limited degree in cartilage obtained from the collateral compartment. Since LIPUS stimulated both chondrocyte proliferation and matrix production by chondrocytes of articular human cartilage in vitro, it might provide a feasible tool for cartilage tissue repair in osteoarthritic patients.

Acknowledgments The study was supported by Exogen (Smith \& Nephew division, Memphis, Tennessee, USA). The authors wish to thank Dr. P. A. Nolte (Spaarne Hospital, The Netherlands) for critically reading the manuscript.

Open Access This article is distributed under the terms of the Creative Commons Attribution Noncommercial License which permits any noncommercial use, distribution, and reproduction in any medium, provided the original author(s) and source are credited.

\section{References}

1. Cohen J (1988) Statistical power analysis for the behavioral sciences, 2nd edn. Lawrence Erlbaum Associates, Inc. Publishers, Mahwah
2. Cook SD, Salkeld SL, Popich-Patron LS, Ryaby JP, Jones DG, Barrack RL (2001) Improved cartilage repair after treatment with low-intensity pulsed ultrasound. Clin Orthop Relat Res 391S:S231-S243. doi:10.1097/00003086-200110001-00022

3. Duda GN, Kliche A, Kleemann R, Hoffmann JE, Sittinger M, Haisch A (2003) Does low-intensity pulsed ultrasound stimulate maturation of tissue-engineered cartilage? J Biomed Mater Res Part B Appl Biomater 68B:21-28. doi:10.1002/jbm.b.10075

4. Ebisawa K, Hata K-I, Okada K, Kimata K, Ueda M, Torii S et al (2004) Ultrasound enhances transforming growth factor $\beta$-mediated chondrocyte differentiation of human mesenchymal stem cells. Tissue Eng 10:921-929. doi:10.1089/1076327041348437

5. Heckman JD, Ryaby JP, McCabe J, Frey JJ, Kilcoyne RF (1994) Acceleration of tibial fracture-healing by non-invasive, lowintensity pulsed ultrasound. J Bone Joint Surg Am 76-A(1):26-34

6. Hunziker EB (2001) Articular cartilage repair: basic science and clinical progress. A review of the current status and prospects. Osteoarthritis Cartilage 10:432-463. doi:10.1053/joca.2002.0801

7. Iorio R, Healey WL (2003) Unicompartimental osteoarthritis of the knee. J Bone Joint Surg Am 85:1351-1364

8. Klein-Nulend J, Veldhuijzen JP, van de Stadt RJ, van Kampen GPJ, Kuijer R, Burger EH (1987) Influence of intermittent compressive force on proteoglycan content in calcifying growth plate cartilage in vitro. J Biol Chem 262(32):15490-15495

9. Kopakkala-Tani M, Leskinen JJ, Karjalainen HM, Karjalainen T, Hynynen K, Töyräs J et al (2006) Ultrasound stimulates proteoglycan synthesis in bovine primary chondrocytes. Biorheology 43:271-282

10. Korstjens CM, Nolte PA, Burger EH, Albers GHR, Semeins CM, Aartman IHA et al (2004) Stimulation of bone cell differentiation by low-intensity ultrasound-a histomorphometric in vitro study. J Orthop Res 22:495-500. doi:10.1016/j.orthres.2003.09.011

11. Kristiansen TK, Ryaby JP, McCabe J, Frey JJ, Roe LR (1997) Accelerated healing of distal radial fractures with the use of specific, low-intensity ultrasound. J Bone Joint Surg Am 79-A(7): 961-973

12. Mukai S, Ito H, Nakagawa $\mathrm{Y}$, Akiyama $\mathrm{H}$, Miyamoto $\mathrm{M}$, Nakamura T (2005) Transforming growth factor-beta1 mediates the effects of low-intensity pulsed ultrasound in chondrocytes. Ultrasound Med Biol 31(12):1713-1721. doi:10.1016/j. ultrasmedbio.2005.07.012

13. Nishikori T, Ochi M, Uchio Y, Maniwa S, Kataoka H, Kawasaki $\mathrm{K}$ et al (2002) Effects of low-intensity pulsed ultrasound on proliferation and chondroitin sulphate synthesis of cultured chondrocytes embedded in Atelocollagen ${ }^{\circledR}$ gel. J Biomed Mater Res 59:201-206. doi:10.1002/jbm.1226

14. Nolte PA, van der Krans A, Patka P, Janssen IGM, Ryaby JP, Albers GHR (2001) Low-intensity pulsed ultrasound in the treatment of nonunions. J Trauma 51:693-703. doi:10.1097/0000 5373-200110000-00012

15. Nolte PA, Klein-Nulend J, Albers GHR, Marti RK, Semeins CM, Goei SW et al (2001) Low-intensity ultrasound stimulates endochondral ossification in vitro. J Orthop Res 19:301-307. doi: 10.1016/S0736-0266(00)00027-9

16. Parvizi J, Wu C-C, Lewallen DG, Greenleaf JF, Bolander ME (1999) Low-intensity ultrasound stimulates proteoglycan synthesis in rat chondrocytes by increasing aggrecan gene expression. J Orthop Res 17:488-494. doi:10.1002/jor.1100 170405

17. Parvizi J, Parpura V, Greenleaf JF, Bolander ME (2002) Calcium signaling is required for ultrasound-stimulated aggrecan synthesis by rat chondrocytes. J Orthop Res 20:51-57. doi:10.1016/ S0736-0266(01)00069-9

18. Pilla AA, Mont MA, Nasser PR, Khan SA, Figueiredo M, Kaufman JJ et al (1990) Non-invasive low-intensity pulsed 
ultrasound accelerates bone healing in the rabbit. J Orthop Trauma 4(3):246-253. doi:10.1097/00005131-199003000-00002

19. Rutten S, Nolte PA, Guit GL, Bouman DE, Albers GHR (2007)

Use of low-intensity pulsed ultrasound for posttraumatic nonunions of the tibia, a review of patients treated in the Netherlands. J Trauma 62(4):902-908. doi:10.1097/01.ta.0000 238663.33796.fb

20. Yang K-H, Parvizi J, Wang S-J, Lewallen DG, Kinnick RR, Greenleaf JF et al (1996) Exposure to low-intensity ultrasound increases aggrecan gene expression in a rat femur fracture model. J Orthop Res 14:800-809. doi:10.1002/jor.1100140518
21. Zhang Z-J, Huckle J, Francomano CA, Spencer RGS (2002) The influence of pulsed low-intensity ultrasound production of chondrocytes at different stages of differentiation: an explant study. Ultrasound Med Biol 28:1547-1553. doi:10.1016/ S0301-5629(02)00659-2

22. Zhang Z-J, Huckle J, Francomano CA, Spencer RGS (2003) The effects of pulsed low-intensity ultrasound on chondrocyte viability, proliferation, gene expression and matrix production. Ultrasound Med Biol 29:1645-1651. doi:10.1016/j.ultrasmedbio. 2003.08.011 